\title{
Solvable and/or integrable many-body models on a circle
}

\author{
Oksana Bihun $^{* 1}$ and Francesco Calogero ${ }^{+2}$ \\ *Department of Mathematics, Concordia College at Moorhead, MN, USA \\ +Physics Department, University of Rome "La Sapienza" and Istituto \\ Nazionale di Fisica Nucleare, Sezione di Roma \\ ${ }^{1}$ obihun@cord.edu \\ ${ }^{2}$ francesco.calogero@roma1.infn.it, francesco.calogero@uniroma1.it
}

\begin{abstract}
Various many-body models are treated, which describe $N$ points confined to move on a plane circle. Their Newtonian equations of motion ("accelerations equal forces") are integrable, i. e. they allow the explicit exhibition of $N$ constants of motion in terms of the dependent variables and their time-derivatives. Some of these models are moreover solvable by purely algebraic operations, by (explicitly performable) quadratures and, finally, by functional inversions. The techniques to manufacture these models are not new; some of these models are themselves new; others are reinterpretations of known models.
\end{abstract}

\section{Introduction}

The investigation of the time evolution of an arbitrary number $N$ of pointparticles the dynamics of which is determined by Newtonian equations of motion ("accelerations equal forces") is of course a fundamental topic in physics and mathematics. The identification in this context of models amenable to exact treatments is a major area of research in mathematical physics and applied mathematics, having a centuries-old history and having been boosted by developments in the last few decades, which also impacted several areas of physics beyond mechanics and many fields of pure mathematics. An interesting related development which is now becoming of interest is the study of such models in which the motion is restricted to lie on an a priori prescribed manifold: see for instance 1 [ 2 [3 [4]. In this paper we make some initial, simple steps in this direction by focussing on various many-body models describing the evolution of $N$ points whose positions on a plane are characterized by $N$ unit 2 -vectors, thereby forcing their motion to be confined to a circle of unit radius centered at the origin. All these models are characterized by Newtonian equations of motion: accelerations equal forces, which in these models are of one-body, two-body or, in some cases, many-body type, and might depend on the velocities of the moving particles in addition to their positions. All these models are autonomous: their equations of motion are time-independent. They 
are all amenable to exact treatments: in particular they all allow the explicit identification of $N$ constants of motion in terms of the $N$ dependent variables and their $N$ time-derivatives (for terminological simplicity we hereafter call such models integrable). In some cases their initial-value problems can be moreover solved by (explicitly performable) quadratures and subsequent functional inversions, preceded by purely algebraic operations, such as solving systems of linear constant-coefficients ODEs, or (equivalently) evaluating the $N$ eigenvalues of known (time-dependent) $N \times N$ matrices or (equivalently) the $N$ zeros of known (time-dependent) polynomials of degree $N$ (for terminological simplicity we hereafter call such models solvable). The techniques to manufacture these models are not new; some of these models are themselves new; others are essentially reinterpretations of known models. The dynamics of these models are not analyzed in detail; but in some cases the main features of their behavior are ascertained, for instance for isochronous models the time evolution of which is isochronous (i. e., completely periodic with a fixed period independent of the initial data), or for models all motions of which are multiply periodic.

The equations of motion of the $N$-body problems treated below are listed with minimal comments in the following Section 2, to facilitate the hasty reader wishing to get an immediate idea of the findings reported in this paper. These results are then proven in the subsequent Section 3: the titles of its subsections indicate case-by-case the techniques employed to arrive at the relevant results. Finally, a terse Section 4 entitled "Outlook" outlines possible developments, to be eventually reported in other papers. Some mathematical details are confined to two Appendices.

\section{Many-body models on a circle amenable to exact treatments}

In the following subsections we display, with minimal comments, various $N$-body problems of Newtonian type ("accelerations equal forces") describing motions on a circle and amenable to exact treatments (detailed in the following Section 3 ). But we provide firstly a terse subsection devoted to notation.

\section{$2.1 \quad$ Notations}

The models under consideration generally feature $N$ points moving in a plane. We identify these $N$ points by 3 -vectors $\vec{r}_{n}, n=1,2, \ldots, N$ for which we use the following 3-dimensional notation:

$$
\vec{r}_{n} \equiv\left(\cos \theta_{n}, \quad \sin \theta_{n}, 0\right) \equiv\left(x_{n}, y_{n}, 0\right) .
$$

Hereafter $N$ is an arbitrary positive integer (generally $N \geq 2$ ) and indices such as $n, m, \ell$ run over the positive integers from 1 to $N$ (unless otherwise explicitly indicated). 
Clearly these vectors $\vec{r}_{n}$ have unit length,

$$
\vec{r}_{n} \cdot \vec{r}_{n}=1 \text {. }
$$

Throughout this paper the dot sandwiched among two vectors denotes the standard scalar product, so that for instance

$$
\vec{r}_{n} \cdot \vec{r}_{m}=\cos \left(\theta_{n}-\theta_{m}\right) .
$$

It is moreover convenient to introduce the unit vector $\hat{z}$ orthogonal to the $x y$ plane,

$$
\hat{z} \equiv(0,0,1)
$$

and to denote by the "wedge" symbol $\wedge$ the standard (3-dimensional) vector product, so that

$$
\begin{gathered}
\hat{z} \wedge \vec{r}_{n}=-\vec{r}_{n} \wedge \hat{z}=\left(-\sin \theta_{n}, \quad \cos \theta_{n}, 0\right), \\
\left(\hat{z} \wedge \vec{r}_{m}\right) \cdot \vec{r}_{n}=\left(\vec{r}_{m} \wedge \vec{r}_{n}\right) \cdot \hat{z}=\sin \left(\theta_{n}-\theta_{m}\right) .
\end{gathered}
$$

Hereafter we deal with time-dependent vectors

$$
\vec{r}_{n}(t) \equiv\left(\cos \theta_{n}(t), \quad \sin \theta_{n}(t), 0\right),
$$

and superimposed dots indicate derivatives with respect to the time variable $t$ so that, for instance,

$$
\begin{aligned}
& \dot{\vec{r}}_{n}=\dot{\theta}_{n}\left(-\sin \theta_{n}, \cos \theta_{n}, 0\right)=\dot{\theta}_{n} \hat{z} \wedge \vec{r}_{n}, \\
\ddot{\vec{r}}_{n}= & \ddot{\theta}_{n}\left(-\sin \theta_{n}, \cos \theta_{n}, 0\right)-\dot{\theta}_{n}^{2}\left(\cos \theta_{n}, \sin \theta_{n}, 0\right) \\
= & \ddot{\theta}_{n} \hat{z} \wedge \vec{r}_{n}-\dot{\theta}_{n}^{2} \vec{r}_{n} .
\end{aligned}
$$

Note that here we omitted, for notational simplicity, to indicate explicitly the time-dependence of the quantities appearing in these $N$ equations; we will often do this below without repeating this warning.

Several other identities are reported in Appendix A: they are useful to obtain the results reported below, but are not necessary to understand the findings reported in the following subsections. 


\subsection{Two models obtained via techniques of generalized La- grangian interpolation}

First model:

$$
\begin{aligned}
& \mu_{n} \overrightarrow{\vec{r}}_{n}=-\mu_{n}\left(\dot{\vec{r}}_{n} \cdot \dot{\vec{r}}_{n}\right) \vec{r}_{n} \\
& +\hat{z} \wedge \vec{r}_{n}\left\{\left[\mu_{n}\left(\dot{\vec{r}}_{n} \cdot \dot{\vec{r}_{n}}\right)+\eta_{n}\left(\vec{r}_{n} \wedge \dot{\vec{r}_{n}}\right) \cdot \hat{z}\right] \sum_{\ell=1, \ell \neq n}^{N}\left[\frac{\left(\vec{r}_{\ell} \cdot \vec{r}_{n}\right)}{\left(\vec{r}_{\ell} \wedge \vec{r}_{n}\right) \cdot \hat{z}}\right]\right. \\
& \left.+\left[\left(\vec{r}_{n} \wedge \dot{\vec{r}_{n}}\right) \cdot \hat{z}\right] \sum_{\ell=1, \ell \neq n}^{N}\left[\frac{\sigma_{n}(\underline{\vec{r}})}{\sigma_{\ell}(\underline{\vec{r}})} \frac{\left.\mu_{\ell}\left(\vec{r}_{\ell} \wedge \dot{\vec{r}}_{\ell}\right) \cdot \hat{z}+\eta_{\ell}\right]}{\left(\vec{r}_{\ell} \wedge \vec{r}_{n}\right) \cdot \hat{z}}\right]\right\} \\
& \sigma_{n}(\underline{\vec{r}})=\prod_{\ell=1, \ell \neq n}^{N}\left[\left(\vec{r}_{\ell} \wedge \vec{r}_{n}\right) \cdot \hat{z}\right] .
\end{aligned}
$$

Second model:

$$
\begin{aligned}
& \mu_{n} \vec{r}_{n}==-\mu_{n}\left(\dot{\vec{r}}_{n} \cdot \dot{\vec{r}}_{n}\right) \vec{r}_{n} \\
& +\sum_{\ell=1, \ell \neq n}^{N}\left\{[ ( \vec { r } _ { \ell } \wedge \vec { r } _ { n } ) \cdot \hat { z } ] ^ { - 1 } \left\{\left[\left(\vec{r}_{n} \wedge \dot{\vec{r}}_{n}\right) \cdot \hat{z}\right]\left[\mu_{\ell}\left(\vec{r}_{\ell} \wedge \dot{r}_{\ell}\right) \cdot \hat{z}+\eta_{\ell}\right]\right.\right. \\
& \left.\left.+\left[\mu_{n}\left(\vec{r}_{n} \wedge \dot{\vec{r}}_{n}\right) \cdot \hat{z}+\eta_{n}\right]\left[\left(\vec{r}_{\ell} \wedge \dot{\vec{r}}_{\ell}\right) \cdot \hat{z}\right]\right\}\left(\vec{r}_{\ell} \wedge \vec{r}_{n}\right)\right\} .
\end{aligned}
$$

In these Newtonian equations $\mu_{n}$ and $\eta_{n}$ are $2 N$ arbitrary constants, and for the rest of the notation see Subsection 2.1; note in particular the property (2a), implying that the $N$ vectors $\vec{r}_{n}$ have unit modulus, hence that the $N$ points whose time evolution is determined by these equations of motion are constrained to move on the circle of unit radius centered at the origin of the Cartesian plane.

These equations of motion are covariant, implying that the corresponding $N$-body problems are rotation-invariant.

These two $N$-body problems are both integrable: they possess $N$ constants of motion, the explicit expressions of which in terms of the vectors $\vec{r}_{n}$ and their

time-derivatives $\vec{r}_{n}$ are displayed in the following Subsection 3.1. The equations of motion of the first, (7a), of these two models feature many-body forces due to the presence in their right-hand ("forces") sides of the quantities $\sigma_{n}(\underline{\vec{r}})$, see (7b), but their initial-value problem is solvable by purely algebraic operations; nevertheless their time evolution can be quite complicated (detailed analyses are not performed in this paper; the fact that solvable models can exhibit quite complicated dynamics is of course well known, see for instance the papers where a 3-body model is studied the time evolution of which is highly nontrivial in spite 
of the fact that its Aristotelian equations of motion - "velocity equal forces" are quite neat and that its initial-value problem can be reduced to solving a single algebraic equation [6]).

\subsection{Two solvable models obtained via a reinterpretation of known models}

The first model is merely a transcription of the solvable "Sutherland model", see Subsection 3.2. It reads as follows:

$$
\ddot{\vec{r}}_{n}=-\left(\dot{\vec{r}}_{n} \cdot \dot{\vec{r}}_{n}\right) \vec{r}_{n}+g^{2} \hat{z} \wedge \vec{r}_{n} \sum_{\ell=1, \ell \neq n}^{N}\left\{\frac{\vec{r}_{n} \cdot \vec{r}_{\ell}}{\left[\left(\vec{r}_{\ell} \wedge \vec{r}_{n}\right) \cdot \hat{z}\right]^{3}}\right\} .
$$

Here $g$ is an arbitrary "coupling constant", and the rest of the notation is, we trust, clear (see Subsection 2.1).

The second model is also merely a transcription of a well-known solvable model ("of goldfish type"), see Subsection 3.2. It reads as follows:

$$
\begin{aligned}
& \ddot{\vec{r}}_{n}=-\left(\dot{\vec{r}}_{n} \cdot \dot{\vec{r}}_{n}\right) \vec{r}_{n}+g_{0} \hat{z} \wedge \vec{r}_{n}+g_{1} \dot{\vec{r}}_{n} \\
& +\hat{z} \wedge \vec{r}_{n} \sum_{\ell=1, \ell \neq n}^{N}\left\{\frac{2 \dot{\vec{r}}_{n} \cdot \dot{\vec{r}}_{\ell}+g_{2}\left[\left(\dot{\vec{r}}_{n} \wedge \vec{r}_{\ell}+\dot{\vec{r}}_{\ell} \wedge \vec{r}_{n}\right) \cdot \hat{z}\right]+g_{3} \vec{r}_{n} \cdot \vec{r}_{\ell}}{\left(\vec{r}_{\ell} \wedge \vec{r}_{n}\right) \cdot \hat{z}}\right\} .
\end{aligned}
$$

Here the 4 constants $g_{0}, g_{1}, g_{2}$ and $g_{3}$ are arbitrary constants, and the rest of the notation is, we trust, clear (see Subsection 2.1).

These equations of motion are covariant, implying that the corresponding $N$-body problems are rotation-invariant.

\subsection{Two $N$-body problems on a circle obtained by changes of dependent variables}

These two solvable models are merely transcriptions of two well-known onedimensional solvable models, see Subsection 3.3. The first model reads as follows:

$$
\begin{aligned}
& \ddot{\vec{r}}_{n}=-\left(\dot{\vec{r}}_{n} \cdot \dot{\vec{r}}_{n}\right) \vec{r}_{n}-\hat{z} \wedge \vec{r}_{n}\left\{2\left[\left(\dot{\vec{r}}_{n} \cdot \dot{\vec{r}}_{n}\right) \frac{y_{n}}{x_{n}}\right]\right. \\
& \left.+4 x_{n} y_{n}-x_{n}^{5} \sum_{\ell=1, \ell \neq n}^{N}\left[\frac{y_{\ell}}{\left(\vec{r}_{\ell} \wedge \vec{r}_{n}\right) \cdot \hat{z}}\right]^{3}\right\} .
\end{aligned}
$$

Here $x_{n} \equiv \cos \theta_{n}$ and $y_{n} \equiv \sin \theta_{n}$ are the two Cartesian components in the plane of the vector $\vec{r}_{n}$, see (1). 
This model is isochronous with period $\pi$,

$$
\vec{r}_{n}(t \pm \pi)=\vec{r}_{n}(t) .
$$

The second model reads as follows:

$$
\begin{aligned}
& \ddot{\vec{r}}_{n}=-\left(\dot{\vec{r}}_{n} \cdot \dot{\vec{r}}_{n}\right) \vec{r}_{n}-\hat{z} \wedge \vec{r}_{n}\left\{2\left[\left(\dot{\vec{r}}_{n} \cdot \dot{\vec{r}}_{n}\right) \frac{y_{n}}{x_{n}}\right]\right. \\
& \left.+x_{n} y_{n}-x_{n} \sum_{\ell=1, \ell \neq n}^{N}\left\{\frac{2+x_{n}^{2} x_{\ell}^{2}}{x_{\ell}\left[\left(\vec{r}_{\ell} \wedge \vec{r}_{n}\right) \cdot \hat{z}\right]}\right\}\right\} .
\end{aligned}
$$

Here $x_{n} \equiv \cos \theta_{n}$ and $y_{n} \equiv \sin \theta_{n}$ are again the two Cartesian components in the plane of the vector $\vec{r}_{n}$, see (1).

All solutions of this model are multiply periodic, see Subsection 3.3.

Note that - in contrast to the equations of motions reported in the two preceding subsections - those displayed herein, (11a) and (12), are not written in covariant fashion, i. e. without any explicit appearance of the Cartesian components $x_{n} \equiv \cos \theta_{n}$ and $y_{n} \equiv \sin \theta_{n}$ of the vector $\vec{r}_{n}$; indeed these equations of motion are not rotation-invariant, or equivalently, they are not invariant for translations along the circle (on which the motions take place due to the constraint (2a)).

\section{Proofs}

In the following subsections we substantiate the findings reported in the preceding Section 2.

\subsection{Solvable and integrable models on the circle manufac- tured via techniques of generalized Lagrangian inter- polation}

In this subsection we employ the technique to manufacture many-body models amenable to exact treatments introduced in [5] (see in particular Chapter 3 of this book, entitled " $N$-body problems treatable via techniques of exact Lagrangian interpolation in spaces of one or more dimensions"). We begin with a terse review of this method, in the specific case of one-dimensional space with an appropriate choice of the set of "seeds" (namely, of the $N$ functions providing the point of departure for the generalized Lagrangian interpolation approach).

The set of seeds we conveniently take as basis for our treatment are the $N$ functions

$$
\begin{aligned}
& \left\{s_{n}(\theta)\right\}_{n=1}^{N}=\{\exp [i(2 n-N-1) \theta]\}_{n=1}^{N} \\
= & \{\exp [i(1-N) \theta], \exp [i(3-N) \theta], \ldots \\
& \ldots \exp [i(N-3) \theta], \exp [i(N-1) \theta]\} .
\end{aligned}
$$


Remark 3.1.1. These exponential functions with imaginary argument are complex, but clearly this set of seeds could be replaced without significant changes by an equivalent set featuring instead sines and cosines of real arguments. The use of exponentials merely facilitates some of the following developments. Likewise the factor 2 in the argument of these functions has been introduced merely to yield neater versions of the equations of motions that will be obtained, see below. The fact that these seeds are invariant under the transformation $\theta \Rightarrow \theta+2 \pi$ suggests to interpret the variable $\theta$ as an angle in the plane.

We then consider a function $f(\theta)$ representable as a linear superposition of these $N$ seeds,

$$
f(\theta)=\sum_{n=1}^{N}\left[h_{n} s_{n}(\theta)\right]
$$

where the $N$ coefficients $h_{n}$ are a priori arbitrary numbers. And we denote with $f_{n}$ the $N$ values that this function takes at the $N$ (arbitrarily assigned) "nodes" $\theta=\theta_{n}$,

$$
f_{n}=f\left(\theta_{n}\right)
$$

and we display the representation of this function in terms of these $N$ values, via the ("generalized Lagrangian interpolation") formula

$$
f(\theta)=\sum_{n=1}^{N}\left[f_{n} q^{(n)}(\theta \mid \underline{\theta})\right] .
$$

The $N$ "interpolational functions" $q^{(n)}(\theta \mid \underline{\theta})$ depend on the variable $\theta$ and on the $N$ nodes $\theta_{n}$ (hence on the $N$-vector having these nodes as its components, hereafter denoted as $\left.\underline{\theta} \equiv\left(\theta_{1}, \theta_{2}, \ldots, \theta_{N}\right)\right)$; they are themselves linear superpositions of the seeds $s_{n}(\theta)$, to insure consistency among (14c) and (14a); and they feature the property

$$
q^{(n)}\left(\theta_{m} \mid \underline{\theta}\right)=\delta_{n m}
$$

to insure consistency among (14c) and (14b) (here and hereafter $\delta_{n m}$ is the Kronecker symbol: $\delta_{n m}=1$ if $n=m, \delta_{n m}=0$ if $\left.n \neq m\right)$.

The explicit representation of these interpolational functions $q^{(n)}(\theta \mid \underline{\theta})$ in terms of the $N$ seeds $s_{n}(\theta)$ and the $N$ nodes $\theta_{n}$ reads [5]

$$
q^{(n)}(\theta \mid \underline{\theta})=\frac{\Delta\left(\theta_{1}, \ldots, \theta_{n-1}, \theta, \theta_{n+1}, \ldots, \theta_{N}\right)}{\Delta\left(\theta_{1}, \ldots, \theta_{N}\right)},
$$

where

$$
\Delta(\underline{\theta})=\left|\begin{array}{cccc}
s_{1}\left(\theta_{1}\right) & s_{2}\left(\theta_{1}\right) & \ldots & s_{N}\left(\theta_{1}\right) \\
s_{1}\left(\theta_{2}\right) & s_{2}\left(\theta_{2}\right) & \ldots & s_{N}\left(\theta_{2}\right) \\
\vdots & \vdots & \ddots & \vdots \\
s_{1}\left(\theta_{N}\right) & s_{2}\left(\theta_{N}\right) & \ldots & s_{N}\left(\theta_{N}\right)
\end{array}\right|
$$


This determinant - with the set of seeds (13) - is of Vandermonde type hence it can be explicitly evaluated, yielding for the interpolational functions the expression

$$
q^{(n)}(\theta \mid \underline{\theta})=s_{1}\left(\theta-\theta_{n}\right) \prod_{\ell=1, \ell \neq n}^{N}\left[\frac{\exp (2 i \theta)-\exp \left(2 i \theta_{\ell}\right)}{\exp \left(2 i \theta_{n}\right)-\exp \left(2 i \theta_{\ell}\right)}\right] .
$$

The next step is to introduce the time variable $t$. As in [5], we assume hereafter that the $N$ seeds $s_{n}(\theta)$ are time-independent; we moreover assume the function $f(\theta)$ to be also time-independent (thereby simplifying the more general treatment of [5]). A time-dependence is only introduced for the nodes $\theta_{n} \equiv \theta_{n}(t)$; indeed they shall be the dependent variables of the dynamical systems we manufacture. Of course the fact that the nodes $\theta_{n}(t)$ evolve over time entails that the values $f_{n}$ taken by the function $f(\theta)$ at these nodes (see (14b)) also evolve over time:

$$
f_{n} \equiv f_{n}(t)=f\left[\theta_{n}(t)\right] .
$$

We then posit a convenient relation among the time evolution of the $N$ nodes $\theta_{n}(t)$ and the time evolution of the $N$ quantities $f_{n}(t)$, by setting

$$
f_{n}(t)=\rho_{n}[\underline{\theta}(t)] \dot{\theta}_{n}(t)+\gamma_{n}[\underline{\theta}(t)] .
$$

Here we introduced the $2 N$ functions $\rho_{n}(\underline{\theta})$ and $\gamma_{n}(\underline{\theta})$ of the $N$ nodes $\theta_{n}$, that will be assigned later at our convenience (but note that we forsake - again, for simplicity - the possibility to assign an explicit time-dependence to these functions, in addition to their dependence on the $N$ nodes).

The next step is to ascertain the time dependence of the $N$ nodes $\theta_{n} \equiv \theta_{n}(t)$ implied by these assignments. To this end we time-differentiate the relation (19), getting the following expressions for the second time-derivatives of the $N$ nodes $\theta_{n} \equiv \theta_{n}(t)$ :

$$
\rho_{n}(\underline{\theta}) \ddot{\theta}_{n}=\dot{f}_{n}-\sum_{m=1}^{N}\left\{\left[\frac{\partial \gamma_{n}(\underline{\theta})}{\partial \theta_{m}}+\frac{\partial \rho_{n}(\underline{\theta})}{\partial \theta_{m}} \dot{\theta}_{n}\right] \dot{\theta}_{m}\right\} .
$$

Our next step is to evaluate the quantity $\dot{f}_{n}$, which (see (18) ) reads

$$
\dot{f}_{n}=\frac{\partial f\left(\theta_{n}\right)}{\partial \theta_{n}} \dot{\theta}_{n}
$$

To evaluate this quantity we can use the finite-dimensional representation of the differential operator, yielding (for functions which are linear superpositions of the seeds $s_{n}(\theta)$, see (14) $)$, the exact formula [5]

$$
\frac{\partial f\left(\theta_{n}\right)}{\partial \theta_{n}}=\sum_{m=1}^{N}\left[D_{n m}(\underline{\theta}) f_{m}\right]
$$


with the $N \times N$ matrix $D$ defined componentwise as follows [5]:

$$
D_{n m}(\underline{\theta})=\frac{\partial q^{(m)}(\theta \mid \underline{\theta})}{\partial \theta} \text { evaluated at } \theta=\theta_{n},
$$

hence in our case (see (13) and (16)) reading

$$
\begin{gathered}
D_{n m}(\underline{\theta})=\delta_{n m} \sum_{\ell=1, \ell \neq n}^{N} \cot \left(\theta_{n}-\theta_{\ell}\right)+\left(1-\delta_{n m}\right) \frac{\sigma_{n}(\underline{\theta})}{\sigma_{m}(\underline{\theta})} \frac{1}{\sin \left(\theta_{n}-\theta_{m}\right)}, \\
\sigma_{n}(\underline{\theta})=\prod_{\ell=1, \ell \neq n}^{N}\left[\sin \left(\theta_{n}-\theta_{\ell}\right)\right] .
\end{gathered}
$$

Note that this definition coincides, via (4b), with (7b).

We therefore conclude that the system (20) yields the following set of $N$ Newtonian equations of motion for the dependent variables $\theta_{n} \equiv \theta_{n}(t)$ :

$$
\begin{aligned}
& \rho_{n}(\underline{\theta}) \ddot{\theta}_{n}=\dot{\theta}_{n}\left[\rho_{n}(\underline{\theta}) \dot{\theta}_{n}+\gamma_{n}(\underline{\theta})\right] \sum_{\ell=1, \ell \neq n}^{N}\left[\cot \left(\theta_{n}-\theta_{\ell}\right)\right] \\
& +\dot{\theta}_{n} \sum_{\ell=1, \ell \neq n}^{N}\left\{\frac{\sigma_{n}(\underline{\theta})}{\sigma_{\ell}(\underline{\theta})} \frac{\left[\rho_{\ell}(\underline{\theta}) \dot{\theta}_{\ell}+\gamma_{\ell}(\underline{\theta})\right]}{\sin \left(\theta_{n}-\theta_{\ell}\right)}\right\} \\
& -\sum_{m=1}^{N}\left\{\left[\frac{\partial \rho_{n}(\underline{\theta})}{\partial \theta_{m}} \dot{\theta}_{n}+\frac{\partial \gamma_{n}(\underline{\theta})}{\partial \theta_{m}}\right] \dot{\theta}_{m}\right\} .
\end{aligned}
$$

Of course to obtain this system of $N$ second-order ODEs we also used (19).

Let us now emphasize that, as a consequence of the way these $N$-body problems have been manufactured, they are integrable. It is indeed plain that the time independence of the function $f(\theta)$ entails (via (14a), (14b) and (19D) the relations

$$
\sum_{m=1}^{N}\left\{h_{m} s_{m}\left[\theta_{n}(t)\right]\right\}=\rho_{n}[\underline{\theta}(t)] \dot{\theta}_{n}(t)+\gamma_{n}[\underline{\theta}(t)] .
$$

Here we have displayed the time-dependence of the various quantities, in order to emphasize the time-independence of the $N$ coefficients $h_{m}$, which can actually be evaluated by solving this system of $N$ linear equations, thereby obtaining (via (16)) the following formulas:

$$
h_{m}=q^{(m)}\left(\vartheta_{m} \mid \underline{\theta}\right), \quad \vartheta_{m} \equiv \frac{i \log \left[\rho_{m}(\underline{\theta}) \dot{\theta}_{m}+\gamma_{m}(\underline{\theta})\right]}{2 m-N-1},
$$

where of course the $N$ nodes $\theta_{m} \equiv \theta_{m}(t)$ and their $N$ time derivatives $\dot{\theta}_{m} \equiv$ $\dot{\theta}_{m}(t)$ can be evaluated at any arbitrary time $t$. It is thus plain that the $N$-body 
systems (24) are integrable for any arbitrary assignment of the $2 N$ functions $\rho_{m}(\underline{\theta})$ and $\gamma_{m}(\underline{\theta})$ of the $N$ dependent variables $\theta_{n}$, with these $N$ quantities $h_{m}$ providing $N$ constants of motion given by explicit (generally nontrivial) expressions in terms of the $N$ nodes $\theta_{n}$ and their $N$ time-derivatives $\dot{\theta}_{n}$.

We are still free to assign the $2 N$ functions $\rho_{n}(\underline{\theta})$ and $\gamma_{n}(\underline{\theta})$. There are two natural choices.

The first one reads simply

$$
\rho_{n}(\underline{\theta})=\mu_{n}, \quad \gamma_{n}(\underline{\theta})=\eta_{n},
$$

with $\mu_{n}$ and $\eta_{n}$ arbitrary constant parameters. It clearly yields (see (24)) an $N$-body system characterized by the following set of Newtonian equations of motion:

$$
\begin{aligned}
& \mu_{n} \ddot{\theta}_{n}=\dot{\theta}_{n}\left(\mu_{n} \dot{\theta}_{n}+\eta_{n}\right) \sum_{\ell=1, \ell \neq n}^{N}\left[\cot \left(\theta_{n}-\theta_{\ell}\right)\right] \\
& +\dot{\theta}_{n} \sum_{\ell=1, \ell \neq n}^{N}\left[\frac{\sigma_{n}(\underline{\theta})}{\sigma_{\ell}(\underline{\theta})} \frac{\left(\mu_{\ell} \dot{\theta}_{\ell}+\eta_{\ell}\right)}{\sin \left(\theta_{n}-\theta_{\ell}\right)}\right] .
\end{aligned}
$$

Here the functions $\sigma_{n}(\underline{\theta})$ of the $N$ nodes $\theta_{m}$ are of course defined by $(23 \mathrm{~b})$.

The second assignment of the $2 N$ functions $\rho_{n}(\underline{\theta})$ and $\gamma_{n}(\underline{\theta})$ is suggested by the structure of the system (24). It reads

$$
\rho_{n}(\underline{\theta})=\mu_{n} \sigma_{n}(\underline{\theta}), \quad \gamma_{n}(\underline{\theta})=\eta_{n} \sigma_{n}(\underline{\theta}),
$$

where again $\mu_{n}$ and $\eta_{n}$ are arbitrary constant parameters and the functions $\sigma_{n}(\underline{\theta})$ are defined as above, see (23b), implying (by logarithmic differentiation)

$$
\frac{\partial \gamma_{n}(\underline{\theta})}{\partial \theta_{m}}=\gamma_{n}(\underline{\theta})\left\{\delta_{n m} \sum_{\ell=1, \ell \neq n}^{N}\left[\cot \left(\theta_{n}-\theta_{\ell}\right)\right]-\left(1-\delta_{n m}\right) \cot \left(\theta_{n}-\theta_{m}\right)\right\} \text {, }
$$

and likewise

$$
\frac{\partial \rho_{n}(\underline{\theta})}{\partial \theta_{m}}=\rho_{n}(\underline{\theta})\left\{\delta_{n m} \sum_{\ell=1, \ell \neq n}^{N}\left[\cot \left(\theta_{n}-\theta_{\ell}\right)\right]-\left(1-\delta_{n m}\right) \cot \left(\theta_{n}-\theta_{m}\right)\right\} .
$$

Thereby the $N$-body system gets characterized by the following, simpler set of Newtonian equations of motion:

$$
\mu_{n} \ddot{\theta}_{n}=\sum_{\ell=1, \ell \neq n}^{N}\left[\frac{\dot{\theta}_{n}\left(\mu_{\ell} \dot{\theta}_{\ell}+\eta_{\ell}\right)+\left(\mu_{n} \dot{\theta}_{n}+\eta_{n}\right) \dot{\theta}_{\ell} \cos \left(\theta_{n}-\theta_{\ell}\right)}{\sin \left(\theta_{n}-\theta_{\ell}\right)}\right]
$$

The differences among these two $N$-body systems, (27) and (30), deserve to be emphasized: the $N$-body model (27) involves many-body forces, due to 
the presence of the functions $\sigma_{n}(\underline{\theta})$ and $\sigma_{\ell}(\underline{\theta})$ in its right-hand ("forces") side; while the $N$-body model (30) only involves two-body forces. Both systems can be integrated once, corresponding to the transition from their $N$ second-order Newtonian equations of motion to the corresponding $N$ first-order ODEs (25a). On the other hand, as we show below, only the first of these two integrable systems is solvable.

Indeed, for the first system (but not for the second!), the $N$ first-order ODEs (25a) are uncoupled, reading simply, via (26),

$$
\mu_{n} \dot{\theta}_{n}=-\eta_{n}+\sum_{m=1}^{N}\left[h_{m} s_{m}\left(\theta_{n}\right)\right],
$$

or, equivalently (see (13))

$\mu_{n} \exp \left[(N+1) i \theta_{n}\right] \dot{\theta}_{n}=-\eta_{n} \exp \left[(N+1) i \theta_{n}\right]+\sum_{m=1}^{N}\left[h_{m} \exp \left(2 m i \theta_{n}\right)\right]$

where the $N$ quantities $h_{n}$ are explicitly known in terms of the $2 N$ initial data $\theta_{n}(0), \dot{\theta}_{n}(0)$ (via (25b), (26) and (17): see Appendix B).

These first-order ODEs can be integrated; we confine the relevant developments to Appendix B.

Although the technique to manufacture these two solvable and integrable $\mathrm{N}$ body problems, (27) and (30), is not new [5], these models are, to the best of our knowledge, themselves new; hence a detailed discussion of the actual behavior of these systems has not yet been done. In the present paper we limit our consideration to pointing out how these models can be reformulated to describe the evolution of $N$ points whose positions on a plane are characterized by $N$ unit 2-vectors $\vec{r}_{n}(t)$, see the notation introduced in Subsection 2.1. To this end one utilizes the formulas (6b), (2b), (4b) and the relevant ones among those conveniently collected in Appendix A. And it is plain that one thereby obtains the two models (7) and (8).

\subsection{Solvable models on the circle manufactured by rein- terpreting known solvable models}

In this section we tersely indicate how to obtain the two models (9) and (10).

The first model obtains from the $N$-body system characterized by the following Newtonian equations of motion (with velocity-independent two-body forces):

$$
\ddot{\theta}_{n}=g^{2} \sum_{\ell=1, \ell \neq n}^{N}\left[\frac{\cos \left(\theta_{n}-\theta_{\ell}\right)}{\sin ^{3}\left(\theta_{n}-\theta_{\ell}\right)}\right] .
$$

Here $g$ is an arbitrary "coupling constant", and the rest of the notation is, we trust, clear.

This is a well-known solvable many-body problem, generally associated with the name of Bill Sutherland, who was the first to show the possibility to treat 
this $N$-body problem by exact methods (originally in a quantal context [7]); its treatment in a classical (Hamiltonian) context is provided in several textbooks, see for instance [8] [5] [9].

It is plain that the model (9) is merely the transcription of this model via the notation of Subsection 2.1.

The second model obtains from the $N$-body system characterized by the following Newtonian equations of motion (with velocity-dependent one-body and two-body forces):

$$
\ddot{\theta}_{n}=g_{0}+g_{1} \dot{\theta}_{n}+\sum_{\ell=1, \ell \neq n}^{N}\left\{\left[2 \dot{\theta}_{n} \dot{\theta}_{\ell}+g_{2}\left(\dot{\theta}_{n}+\dot{\theta}_{\ell}\right)+g_{3}\right] \cot \left(\theta_{n}-\theta_{\ell}\right)\right\} \text {. }
$$

Here $g_{0}, g_{1}, g_{2}$ and $g_{3}$ are 4 arbitrary coupling constants, and we again trust the rest of the notation to be clear.

This is also a well known solvable model, see for instance eq. (2.3.5-12) on page 199 of $[5]$.

And it is again plain that the model (10) is merely the transcription of this model via the notation of Subsection 2.1 and Appendix A.

\subsection{How to manufacture $N$-body problems with angles as dependent variables}

In the preceding subsection we have shown how certain $N$-body models with dependent variables naturally interpretable as angles can be reformulated as $N$ body models describing the time evolution on a plane of particles constrained to move on a circle. In this subsection we indicate how, via a simple change of dependent variables, essentially any $N$-body model can be reformulated so that its dependent variables can be interpreted as angles, hence subsequently it can also be reformulated (in fact in many ways) so that it describes the time evolution of particles constrained to move on a plane circle.

The trick to achieve this goal is quite elementary and general; we illustrate it below via two examples.

Consider an $N$-body model in which the positions of the $N$ point-particlesmoving in one-dimensional space - are identified by $N$ coordinates $z_{n} \equiv z_{n}(t)$, and perform the change of dependent variables by positing, say,

$$
z_{n}(t)=\tan \left[\theta_{n}(t)\right]
$$

Remark 3.3.1. Of course this assignment defines $\theta_{n}(t)$ only $\bmod (\pi)$; and clearly many other assignments could be instead made - different but having an analogous effect, such as $z_{n}=1 / \sin \left(2 \theta_{n}\right)$, or $z_{n}=\tan ^{3} \theta_{n}$, etc. .

In the first example we take as point of departure the $N$-body problem characterized by the Newtonian equations of motion

$$
\ddot{z}_{n}=-4 z_{n}+g^{2} \sum_{\ell=1, \ell \neq n}^{N}\left[\left(z_{n}-z_{\ell}\right)^{-3}\right] \text {. }
$$


Here $g$ is an arbitrary (real) coupling constant. This is a well-known solvable model (see for instance [5]); it is isochronous, all its solutions being completely periodic with period $\pi$,

$$
z_{n}(t \pm \pi)=z_{n}(t) .
$$

Via the change of dependent variables (34) the equations of motion (35a) become (as the diligent reader will easily verify, utilizing if need be the identities reported in the last part of Appendix A)

$$
\begin{aligned}
& \ddot{\theta}_{n}=-2 \dot{\theta}_{n}^{2} \tan \theta_{n}-4 \sin \theta_{n} \cos \theta_{n} \\
& +g^{2} \sum_{\ell=1, \ell \neq n}^{N}\left[\frac{\cos ^{5} \theta_{n} \sin ^{3} \theta_{\ell}}{\sin ^{3}\left(\theta_{n}-\theta_{\ell}\right)}\right] .
\end{aligned}
$$

Remark 3.3.2. This model of course hereditates the property of isochrony of the model (35a) it has been obtained from:

$$
\theta_{n}(t \pm \pi)=\theta_{n}(t) \quad \bmod (\pi) .
$$

The next task is to transform these equations of motion, (36a), into equations of motion for points moving in the plane but constrained to stay on a circle of unit radius centered at the origin. To realize this goal one may now use the change of dependent variables from the angles $\theta_{n}$ to the vectors $\vec{r}_{n}$ described in Subsection 2.1, using if need be the identities reported in the first part of Appendix A. And it is plain that in this manner one arrives at the equations of motion (11a).

In the second example we take as point of departure the well-known solvable $N$-body problem characterized by the following Newtonian equations of motion (see eq. $(2.3 .4 .2-1)$ on page 188 of [5]):

$$
\ddot{z}_{n}=-z_{n}+\sum_{\ell=1, \ell \neq n}^{N}\left(\frac{2 \dot{z}_{n} \dot{z}_{\ell}+1}{z_{n}-z_{\ell}}\right) .
$$

All solutions of this model are multiply periodic, being (generally nonlinear) superpositions of the $N$ functions $b_{m}(t)=\cos \left(\sqrt{m} t+\beta_{m}\right), m=1, \ldots, N$ (with the $N$ phases $\beta_{m}$ depending on the initial data); for special initial data only functions $b_{m}(t)$ with $m$ a squared-integer contribute, yielding solutions completely periodic with period $2 \pi$. [5]

Via the change of dependent variables (34) equations of motion (37) become (as the diligent reader will easily verify, utilizing again, if need be, the identities reported in the last part of Appendix A)

$$
\begin{aligned}
& \ddot{\theta}_{n}=-2 \dot{\theta}_{n}^{2} \tan \theta_{n}-\sin \theta_{n} \cos \theta_{n} \\
& +\cos \theta_{n} \sum_{\ell=1, \ell \neq n}^{N}\left[\frac{2 \dot{\theta}_{n} \dot{\theta}_{\ell}+\cos ^{2} \theta_{n} \cos ^{2} \theta_{\ell}}{\cos \theta_{n} \sin \left(\theta_{n}-\theta_{\ell}\right)}\right] .
\end{aligned}
$$


Then we transform these equations of motion into equations of motion for points moving in the plane but constrained to stay on a circle of unit radius centered at the origin, by using again the change of dependent variables from the angles $\theta_{n}$ to the vectors $\vec{r}_{n}$ described in Subsection 2.1 via-if need be-the identities reported in the first part of Appendix A. And it is plain that in this manner one arrives at the equations of motion (12).

\section{Outlook}

Our original motivation to undertake this line of research was the intention to manufacture $N$-body problems amenable to exact treatments describing motions on a sphere, or more generally on manifolds. We consider the results reported in this paper as a modest first step in that direction. We also believe that the actual behavior of the new models reported in this paper - see (7) and (8) - shall eventually deserve a more detailed scrutiny than that provided in Subsection 3.1 .

\section{Appendix A: identities}

It is plain that the notation introduced in Subsection 2.1 entails the following additional identities:

$$
\begin{aligned}
& \vec{r}_{n} \cdot \vec{r}_{n}=0, \quad \vec{r}_{n} \cdot \dot{\vec{r}}_{n}=\dot{\theta}_{n}^{2}, \quad\left(\vec{r}_{n} \wedge \dot{r}_{n}\right) \cdot \hat{z}=\dot{\theta}_{n} \\
& \ddot{\vec{r}}_{n} \cdot \vec{r}_{n}=-\dot{\theta}_{n}^{2}, \quad \ddot{\vec{r}}_{n} \cdot\left(\hat{z} \wedge \vec{r}_{n}\right)=\ddot{\theta}_{n}, \\
& \dot{\vec{r}}_{n} \cdot \vec{r}_{m}=-\dot{\theta}_{n} \sin \left(\theta_{n}-\theta_{m}\right) \\
& \dot{\vec{r}}_{n} \cdot \dot{\vec{r}}_{m}=\dot{\theta}_{n} \dot{\theta}_{m} \cos \left(\theta_{n}-\theta_{m}\right) ; \\
& \hat{z} \wedge \dot{\vec{r}}_{n}=-\dot{\theta}_{n} \vec{r}_{n} \\
& \hat{z} \wedge \ddot{\vec{r}}_{n}=-\ddot{\theta}_{n} \vec{r}_{n}-\dot{\theta}_{n}^{2} \hat{z} \wedge \vec{r}_{n} \\
& \left(\dot{\vec{r}}_{n} \wedge \vec{r}_{m}\right) \cdot \hat{z}=-\dot{\theta}_{n} \cos \left(\theta_{n}-\theta_{m}\right), \\
& \left(\dot{\vec{r}}_{n} \wedge \dot{\vec{r}}_{m}\right) \cdot \hat{z}=-\dot{\theta}_{n} \dot{\theta}_{m} \sin \left(\theta_{n}-\theta_{m}\right) .
\end{aligned}
$$

We also display here some relations among the time-dependent "coordinates"

$$
z_{n} \equiv z_{n}(t)=\tan \theta_{n}(t)
$$


and the "angles" $\theta_{n} \equiv \theta_{n}(t)$ :

$$
\begin{gathered}
z_{n}-z_{m}=\frac{\sin \left(\theta_{n}-\theta_{m}\right)}{\cos \theta_{n} \cos \theta_{m}}, \quad \frac{1}{z_{n}-z_{m}}=\frac{\cos \theta_{n} \cos \theta_{m}}{\sin \left(\theta_{n}-\theta_{m}\right)} ; \\
\dot{z}_{n}=\frac{\dot{\theta}_{n}}{\cos ^{2} \theta_{n}}, \quad \dot{z}_{n} z_{m}=\frac{\dot{\theta}_{n} \sin \theta_{m}}{\cos ^{2} \theta_{n} \cos \theta_{m}}, \quad \dot{z}_{n} \dot{z}_{m}=\frac{\dot{\theta}_{n} \dot{\theta}_{m}}{\cos ^{2} \theta_{n} \cos ^{2} \theta_{m}} ; \\
\frac{\dot{z}_{n}+\dot{z}_{m}}{z_{n}-z_{m}}=\frac{\dot{\theta}_{n} \cos ^{2} \theta_{m}+\dot{\theta}_{m} \cos ^{2} \theta_{n}}{\cos \theta_{n} \cos \theta_{m} \sin \left(\theta_{n}-\theta_{m}\right)}, \\
\frac{\dot{z}_{n} z_{m}+\dot{z}_{m} z_{n}}{z_{n}-z_{m}}=\frac{\dot{\theta}_{n} \sin \theta_{m} \cos \theta_{m}+\dot{\theta}_{m} \sin \theta_{n} \cos \theta_{n}}{\cos \theta_{n} \cos \theta_{m} \sin \left(\theta_{n}-\theta_{m}\right)}, \\
\frac{\dot{z}_{n} \dot{z}_{m}}{z_{n}-z_{m}}=\frac{\dot{\theta}_{n} \dot{\theta}_{m}}{\cos \theta_{n} \cos \theta_{m} \sin \left(\theta_{n}-\theta_{m}\right)} ; \\
\ddot{z}_{n}=\frac{\ddot{\theta}_{n}}{\cos ^{2} \theta_{n}}+\frac{2 \dot{\theta}_{n}^{2} \sin \theta_{n}}{\cos ^{3} \theta_{n}}=\frac{\ddot{\theta}_{n}+2 \dot{\theta}_{n}^{2} \tan \theta_{n}}{\cos ^{2} \theta_{n}} .
\end{gathered}
$$

\section{Appendix B: solution of the system (31)}

In this Appendix we indicate how the initial-value problem of the system of $N$ (decoupled) first-order ODEs (31) is solved.

Let us, for notational convenience, make here the following change of variables:

$$
\zeta_{n}(t)=\exp \left[i \theta_{n}(t)\right],
$$

entailing

$$
\dot{\zeta}_{n}(t)=i \dot{\theta}_{n}(t) \exp \left[i \theta_{n}(t)\right] .
$$

We then use the relation (46a) to rewrite the equations of motion (31) as follows:

$$
\mu \zeta^{N} \dot{\zeta}=i\left[-\eta \zeta^{N+1}+\sum_{m=1}^{N}\left(h_{m} \zeta^{2 m}\right)\right] .
$$

Remark B.1. Let us emphasize that, in the last formula and below (in this Appendix B), as a notational simplification, we omit to indicate explicitly the time-dependence of the dependent variable $\zeta_{n} \equiv \zeta_{n}(t)$, as well as its dependence on the index $n$; and likewise the dependence on this index $n$ of the parameters $\mu_{n}$ and $\eta_{n}$.

The ODE (47) can clearly be solved by the following quadrature:

$$
\int_{\zeta(0)}^{\zeta(t)} d \xi \xi^{N-2}\left\{-\eta \xi^{N-1}+\sum_{m=1}^{N}\left[h_{m} \xi^{2(m-1)}\right]\right\}^{-1}=\frac{i t}{\mu} .
$$


To perform the integration it is convenient to introduce the $2(N-1)$ zeros $\xi_{j}$ of the polynomial of degree $2(N-1)$ appearing in the denominator of the integrand,

$$
-\eta \xi^{N-1}+\sum_{m=1}^{N}\left[h_{m} \xi^{2(m-1)}\right]=h_{N} \prod_{j=1}^{2(N-1)}\left(\xi-\xi_{j}\right),
$$

and then the $2(N-1)$ "residues" $\phi_{j}$ defined by setting

$$
\left\{-\eta \xi^{N-1}+\sum_{m=1}^{N}\left[h_{m} \xi^{2(m-1)}\right]\right\}^{-1}=h_{N}^{-1} \sum_{j=1}^{2(N-1)}\left(\frac{\phi_{j}}{\xi-\xi_{j}}\right) .
$$

Note that these formulas imply that the computation of, firstly, the $2(N-1)$ zeros $\xi_{j}$, and, secondly, the $2(N-1)$ residues $\phi_{j}$, is a purely algebraic task (although not one that can be analytically performed for $N \geq 3$ ); hence these quantities can in principle be considered known functions of the parameter $\eta$ (from which they inherit a dependence on the index $n$, see Remark B.1) and of the $N$ constants of motion $h_{m}$. As for these $N$ quantities $h_{m}$ (which are of course independent of the index $n$ ) they are - in the context of the initial-value problem for the dynamical system (27) - explicitly given by the formulas (25b) at $t=0$ (let us reiterate that these expressions of the $N$ constants of motion $h_{m}$ are valid throughout the time evolution, and of course, in particular, at the initial time $t=0$ ).

The final step is to perform the integration in the left-hand side of (48). Via (49b) the key ingredient to do so is the formula

$$
\begin{aligned}
& \int_{\zeta_{0}}^{\zeta} d \xi \frac{\xi^{N-2}}{\xi-\xi_{0}}=\int_{\zeta_{0}-\xi_{0}}^{\zeta-\xi_{0}} d \xi \frac{\left(\xi+\xi_{0}\right)^{N-2}}{\xi} \\
= & \int_{\zeta_{0}-\xi_{0}}^{\zeta-\xi_{0}} d \xi \sum_{k=0}^{N-2}\left[\left(\begin{array}{c}
N-2 \\
k
\end{array}\right) \xi^{k-1} \xi_{0}^{N-2-k}\right] \\
= & \xi_{0}^{N-2} \log \left(\frac{\zeta-\xi_{0}}{\zeta_{0}-\xi_{0}}\right)+\sum_{k=1}^{N-2}\left\{\left(\begin{array}{c}
N-2 \\
k
\end{array}\right) \frac{\xi_{0}^{N-2-k}}{k}\left[\left(\zeta-\xi_{0}\right)^{k}-\left(\zeta_{0}-\xi_{0}\right)^{k}\right]\right\} .
\end{aligned}
$$

\section{References}

[1] L. Auslander and L. Markus, "Classical differential equations on manifolds", Trans. Amer. Math. Soc. 91, 113-128 (1959).

[2] P. E. Crouch and R. Grossman, "Numerical integration of ordinary differential equations on manifolds", J. Nonlinear Sci. 3, 1-33 (1993). 
[3] E. Hairer, Solving Differential Equations on Manifolds, Lecture Notes, Université de Genève, 2011.

[4] F. Erman and O. T. Turgut, "A many-body problem with point-interactions on two-dimensional manifolds", arXiv:1204.2171v2[math-ph]12Apr2012.

[5] F. Calogero, Classical Many-Body Problems Amenable to Exact Treatments, Lecture Notes in Physics Monographs m66, Springer, Berlin, 2001.

[6] F. Calogero, D. Gómez-Ullate, P. M. Santini and M. Sommacal, "The transition from regular to irregular motions, explained as travel on Riemann surfaces", J. Phys. A: Math. Gen. 38, 8873-8896 (2005); "Towards a theory of chaos explained as travel on Riemann surfaces", J. Phys. A.: Math. Theor. 42, 015205 (26 pages) (2009); D. Gómez-Ullate, P. M. Santini, M. Sommacal and F. Calogero, "Understanding complex dynamics by means of an associated Riemann surface", Physica D 241, 1291-1305 (2012).

[7] B. Sutherland, "Exact results for a quantum many-body problem in one dimension", Phys. Rev. A4, 2019-2021 (1971); "Exact results for a quantum many-body problem in one dimension. II", Phys. Rev. A5, 1372-1376 (1972).

[8] A. M. Perelomov, Integrable systems of classical mechanics and Lie algebras, Birkhäuser, Basel, 1990.

[9] B. Sutherland, Beautiful models - 70 years of exactly solved quantum manybody problems, World Scientific, Singapore, 2004. 